\title{
Design of an Intelligent PH Sensor for Aquaculture Industry
}

\author{
Haijiang Tai ${ }^{1}$, Qisheng Ding ${ }^{1,2, *}$, Daoliang $\mathrm{Li}^{1, * *}$, and Yaoguang $\mathrm{Wei}^{1}$ \\ ${ }^{1}$ College of Information and Electrical Engineering, China Agricultural University, \\ Beijing 100083, P.R. China \\ dliangl@cau.edu.cn \\ ${ }^{2}$ Xuzhou Normal University, Xuzhou, Jiangsu, P.R. China
}

\begin{abstract}
Automatic monitoring and controlling system is essential for improvement of aquaculture industry. This paper presents an intelligent $\mathrm{PH}$ sensor that applied to water quality monitoring. It involved signal processing, self-calibration. Using low power and high performance microcontroller MSP430F149, Smart Transducer Interface Module (STIM) based on IEEE1451standard was embedded. The design of software and hardware was described in detail, and an initial calibration experiment was carried out to establish a calibration parameter. The results indicate that the $\mathrm{pH}$ sensor is more accurate, reliable and easy to use, which is suitable to spread the application in aquaculture industry.
\end{abstract}

Keywords: Intelligent sensor, IEEE1451, PH value.

\section{Introduction}

Over the past decade, the development and applications of chemical sensors and biosensors have grown rapidly. Among all sensors, $\mathrm{pH}$ sensors have received the most attention because of the importance of $\mathrm{pH}$ measurement in various scientific research and practical applications. China's water quality online automatic monitoring technology is laggard; a lot of monitoring methods are still traditional, testing data lags behind seriously. Monitoring instrument water quality auto-monitoring system mainly use 2-wire transducers, exist amount of problem, such as poor stability of signal, high cost, single function, can't connect with field bus control system(Xu hao, 2007).

Intelligent of water quality monitoring instrument is important to improve the level and production mode of aquiculture, as a result, amount of intelligent monitoring instruments were developed, more perfectly water quality monitoring instrument were sold by America, Japan, Italy, and so on (Fan Xiang-guo, 2005); at present, water quality monitoring instrument have rapid development in China, various $\mathrm{PH}$

\footnotetext{
Qisheng Ding, contributed equality with Haijiang Tai, and they are co-first author.

*** Corresponding author.
} 
monitoring instruments developed by Tsinghua University (Fu Xiao-shi et al., 2004; Gong Yuan-ming et al., 2003), Southeast University (Chen Ming-wei et al., 2005), Jiangsu University (Liu Xing-qiao et al., 2006), and so on. The above water quality PH monitoring instrument bases on MCU generally, monitor single or multiple water quality parameters, achieved electrode calibration and Temperature compensation by using software, but it is difficult to connect with field bus control system (Yang Su-ying et al., 2004). Some instruments can connect with field bus control system, but the problem of the interface and incompatibility are generally exist between multi-sensor and actuator (L.Camara, 2004; Institute of Electrical, 1997; Guangming Song et al., 2005; Antonio Pardo, 2006).

In this paper, an intelligent PH sensor was developed according to IEEE145 standard, read sensor data and set actuator parameter by using Transducers Electronic Data Sheets (TEDS) so as to achieved "Plug and play" function (Miroslav S. and Radimir V, 2003). Water temperature and $\mathrm{pH}$ can be measured on-line by the intelligent $\mathrm{pH}$ sensor.

\section{Hardware of the Sensor}

\subsection{Hardware Structure}

The sensor mainly contained signal conditioning model, A/D circuit, power source model, microprocessor and Transducers Electronic Data Sheets (TEDS), Hardware structure of the $\mathrm{pH}$ sensor is shown in Fig. 1.

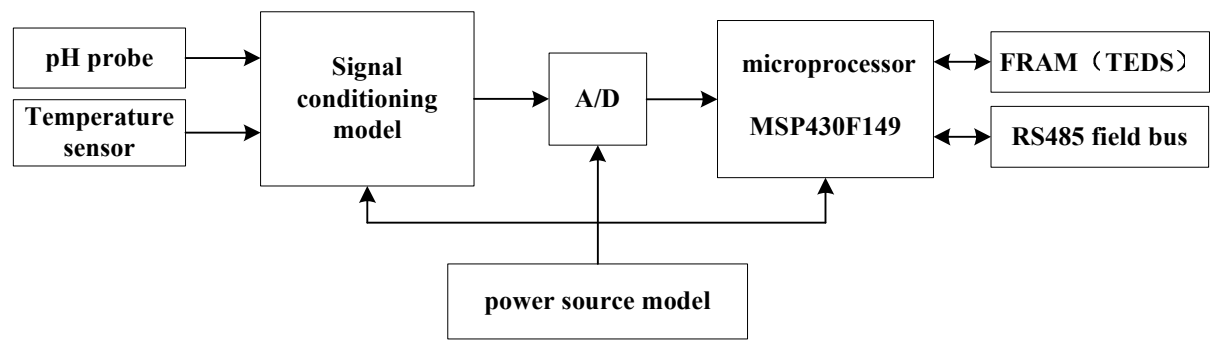

Fig. 1. Hardware structure of $\mathrm{pH}$ sensor

\subsection{Hardware Circuit}

Microcontroller of the sensor is based on MSP430F149 manufactured by TI Company (U.S.A.). The sensor unite adopt an industrial on-line sensor HG990pH and digital temperature sensor DS18B20.

Conversion of the current and amplification of the voltage is achieved by using three high impedance, low drift operational amplifiers TLC27L4; two of them make up differential amplifier, and its input resistance up to $3 \times 1012 \mathrm{ohm}$, to ensure the input 
signal not affected by resistance change. Moreover, differential circuit have high CMRR, can adapt to complex Industry-environment.

In order to meet the market demand, and meet the requirement of remote transmission and networking, the sensor uses RS-485 field bus to make connection and achieve the communication between CPU and Remote I/O by using a SN75LBC184 RS-232/RS-485 convertor. Two resistors (20ohm in this work), R1 and R5, were series connected to the output terminal of SN75LBC184, avoid network failures of a single hardware device affect network. The electric circuit used to drive the sensor and to treat the signal is shown in Fig. 2.

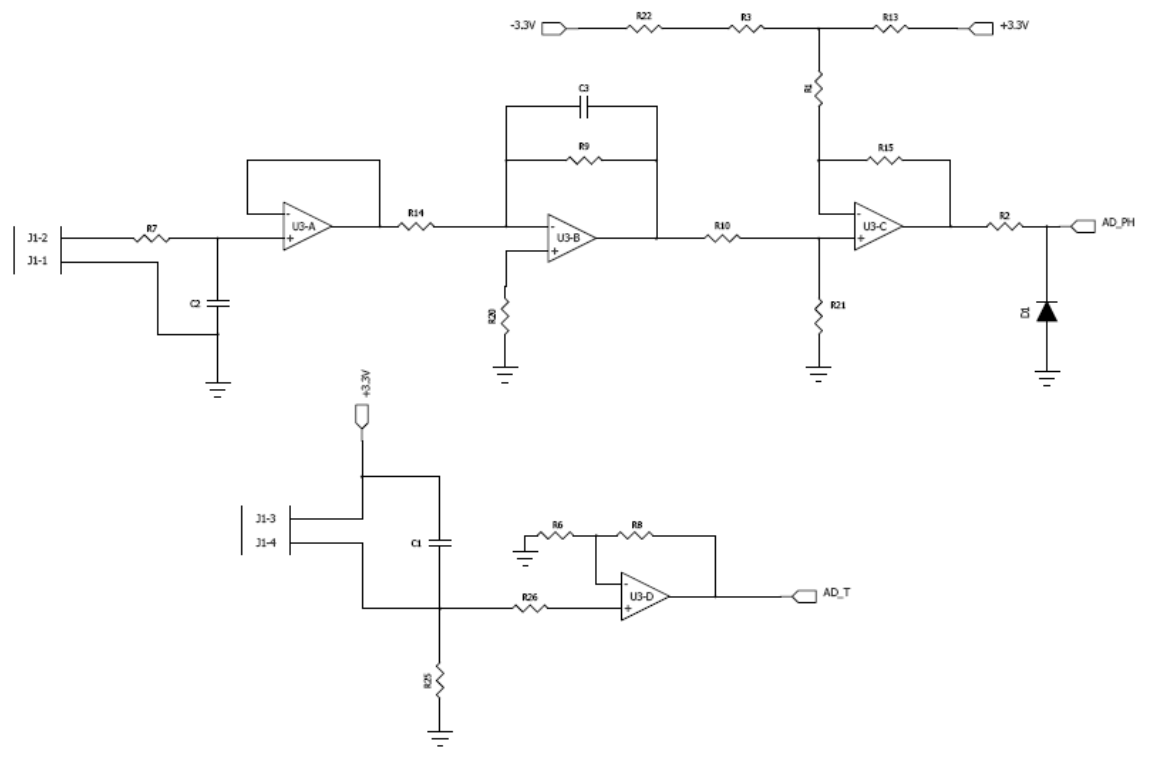

Fig. 2. Electric circuit for the sensor signal treatment

\section{Software of the Sensor}

\subsection{STIM Master Control Program}

STIM master control program include (1) finish the initialization of hardware; (2) loading TEDS information; (3) finish self-configuring of STIM Module; (4) setting various special register; (5) initialize working mode for UART; (6) setting working mode for timer and correlative variables; (7) definition of STIM channel.

STIM master control program adopt circle inquire, initialize the system after power-on reset, then query data buffer to judge if the command is coming. The management of buffers is achieved by sensor address and function module. If data buffer have data coming, analyze it; if the data is addressing command, call address processing module; if the data is management command of TEDS, call TEDS module. Flow chart of STIM master control program is shown in Fig.3. 


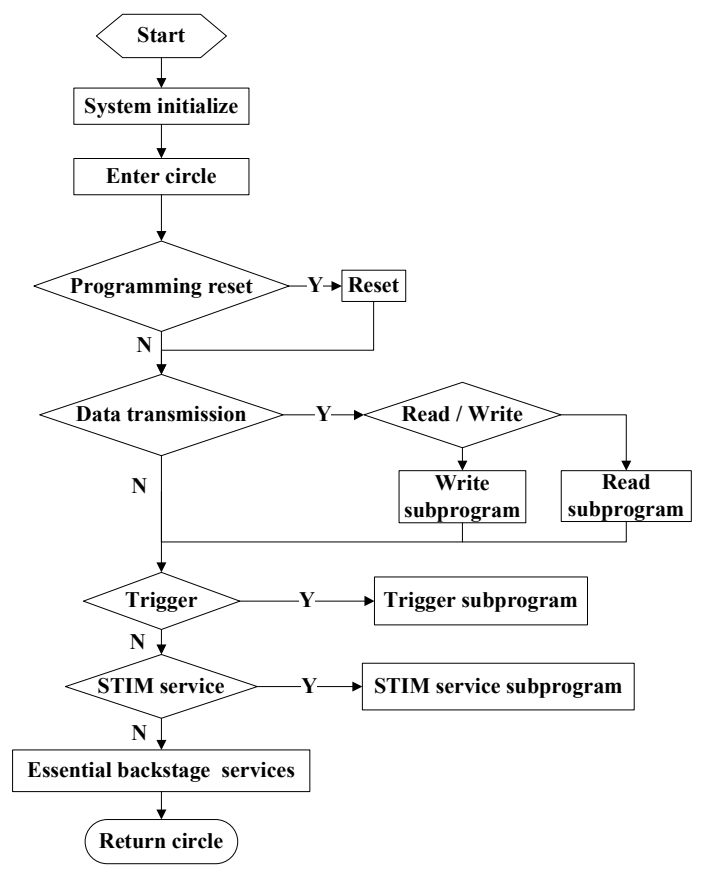

Fig. 3. Flowchart of software for STIM

\subsection{Design of TEDS}

In order to realize the plug and play functionality and self-tuning functionality, Meta-TEDS and Channel-TEDS of TEDS is necessary, besides, Calibration-TEDS and Meta-ID TEDS are also be adopted.

STIM module include one Meta-TEDS, two Channel-TEDS which used to store temperature data and input signal of PH sensor, one Calibration-TEDS and one Meta-ID TEDS. The basic design of TEDS is shown in Tab. 1.

Table 1. The basic design of TEDS

\begin{tabular}{llll}
\hline Meta-TEDS & Channel-TEDS & Calibration-TEDS & $\begin{array}{l}\text { Meta-ID } \\
\text { TEDS }\end{array}$ \\
\hline Data & Channel & Latest adjust & Developer \\
length & number & date & name \\
Data & Physics & Adjust & Development \\
style & unit & interval & date \\
STIM & Sensor serial & Adjust & Produce \\
Channel & number & parameter & site \\
number & Sensor & Adjust & Version \\
Using time & style & model & number \\
limit & & &
\end{tabular}




\subsection{Plug and Play Function and Automatic Recognition of STIM}

Plug and play function and automatic recognition are remarkable features of IEEE1451 intelligent sensor, its implementation principle lie in standard of TEDS was recommended. The table stores all the information about sensor style, physics unit, data style, adjust model and developer ID that corresponding sensor channel. By reading TEDS during use, all the information of STIM channel can be obtained, finish the plug and play functionality. Flow chart of plug and play functionality and automatic recognition is shown in Fig.4.

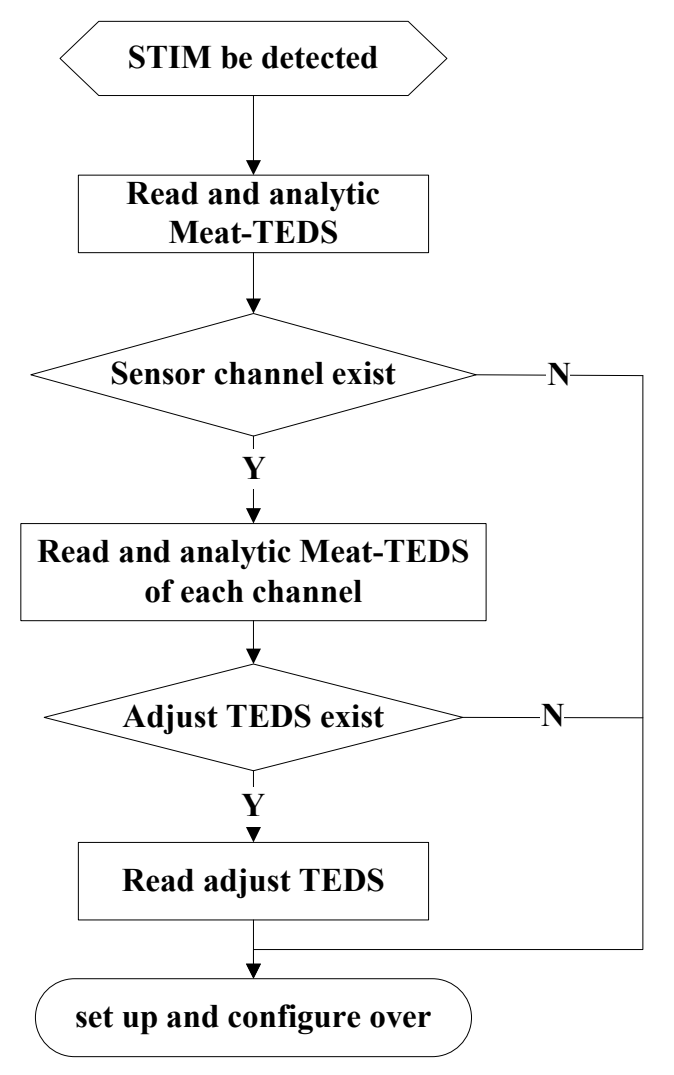

Fig. 4. Flowchart of software for plug-and-play and self-identification

\subsection{Self-correcting}

$\mathrm{N}$ different gauge point in the range of the $\mathrm{PH}$ sensor electronic scale were defined, and $\mathrm{M}$ different gauge point in the range of the temperature were defined, standard input 
value of each gauge point come from standard value generator of $\mathrm{PH}(\mathrm{Y})$ and temperature $(\mathrm{T})$ respectively as follow:

$$
\begin{aligned}
& \mathrm{Y}_{\mathrm{i}}: \mathrm{Y}_{1}, \mathrm{Y}_{2} \ldots, \mathrm{Y}_{\mathrm{N}} \\
& \mathrm{T}_{\mathrm{j}}: \mathrm{T}_{1}, \mathrm{~T}_{2} \ldots, \mathrm{T}_{\mathrm{M}}
\end{aligned}
$$

Read the corresponding output values $\mathrm{U}$ i and $\mathrm{U}$ ii that corresponds to standard input value of each gauge point above mentioned. In this way, the $\mathrm{PH}$ sensor calibrating in static states was done at $\mathrm{M}$ different temperature states, $\mathrm{M}$ different corresponding input and output character cluster ( $\mathrm{Y}-\mathrm{U})$ were obtained. At the same time, $\mathrm{N}$ different input and output character cluster $\left(\mathrm{T}-\mathrm{U}_{\mathrm{T}}\right)$ of temperature sensor corresponds to different PH states were obtained too.

Calculate the correction coefficient of the two character cluster above mentioned by the least square method; deduce the correction equation of intelligent PH sensor. The correction equation stored in Calibration-TEDS. PH value and temperature signal voltage converts into $\mathrm{PH}$ project value after colleted by the microcontroller by using the correction equation stored in Calibration-TEDS, finish the real-time self calibration of $\mathrm{PH}$ and temperature sensor signal measurement errors.

\section{Experiment}

The sensor probe used in this work is based on composite glass electrode HG990 manufactured by Shanghai leici instrument Inc.

The effect caused by temperature was measured by using $\mathrm{PH}$ standard buffer solutions at $\mathrm{pH}$ 4.00, 7.00 and 9.00 manufactured by American HACH Company under the range of $0 \sim 40^{\circ} \mathrm{C}$.

Calibration experiment did by using $\mathrm{PH}$ standard buffer solutions at $\mathrm{pH} 4.00,6.93$ and 9.41 manufactured by American $\mathrm{HACH}$ Company. Experiments were carried out at room temperature, and the measured temperature of standard buffer solution at $(26.7 \pm 0.1)^{\circ} \mathrm{C} . \mathrm{Six}$ measurements at $5 \mathrm{~min}$ intervals were recorded for each standard solution to test reproducibility of the sensor probe by immersing the probe in the solution and lasts $5 \mathrm{~min}$, and then took the probe out after the sensor put out stable signal.

\section{Results and Discussion}

Fig.5 shows the results of temperature effect. As can be seen, no significant temperature effect in the three $\mathrm{PH}$ standard buffer solutions. 


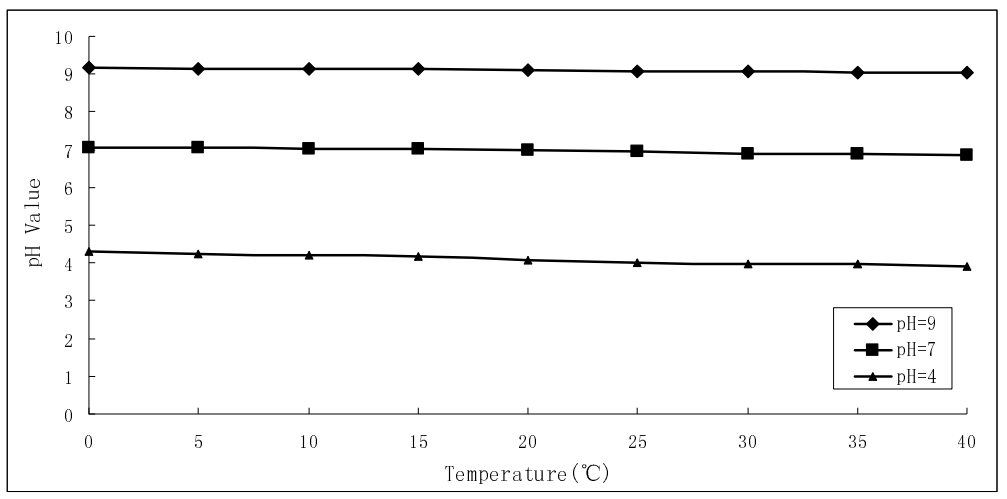

Fig. 5. Temperature effect of an intelligent $\mathrm{pH}$ sensor

Fig. 6 shows the results, voltage changed collected by the sensor for six times below 0.003 , the results shows very high reproducibility of the probe and intelligent $\mathrm{PH}$ sensor designed in this paper. Calibration curve of the probe fitted according to the principle of least square is $y=4.6667 \mathrm{x}+3.1084$, and the correlation coefficient is $\mathrm{R}^{2}=0.9999$, this result shows high Linearity of the probe. Store the calibration curve into Calibration-TEDS, the signal voltage collected by the microcontroller MSP430 converts into PH project value directly, and put out by RS-485 field bus, finish self-calibration, achieve convenience use.

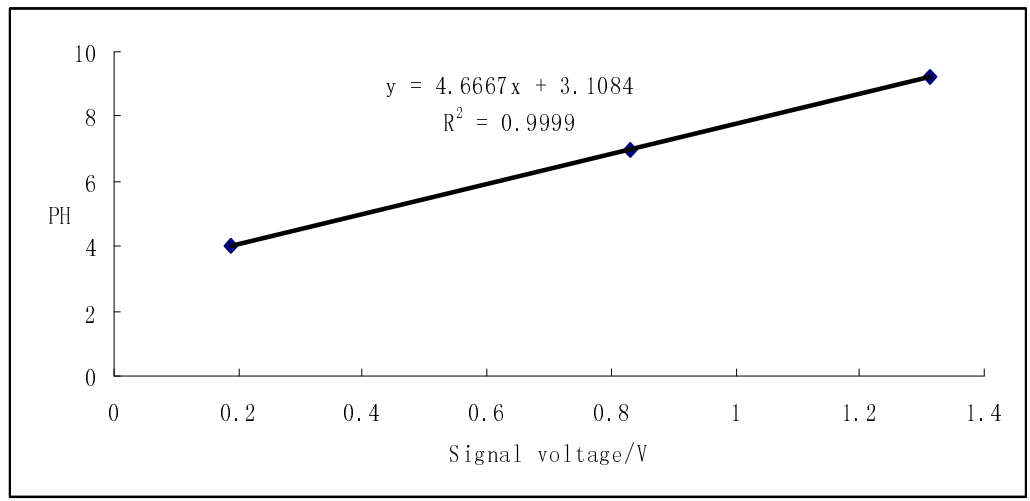

Fig. 6. Calibration results of an intelligent $\mathrm{pH}$ sensor

\section{Conclusion}

The intelligent PH sensor based on IEEE1451standard combined traditional sensor, microcontroller and TEDS technology, thus, the sensor was more intelligent. Concrete representations as following: 
(1) Transmit data by applying all- digital bus, easy to apply in monitoring network;

(2) Intelligent sensor interface module based on IEEE1451 standard achieved plug and play functionality;

(3) Simplified the hardware structures by combining MCU and electrochemistry test technology, expanded measurement function, reduced the cost, improved reliability and measurement precision;

(4) Achieved low power consumption by using low power cost MCU (msp430).

Acknowledgements. This work was supported by 863 programs "Research and Application of Digital Integrated System for Intensive Aquaculture" (2007AA10Z238), 948 Project of China Agriculture Ministry (2010-Z13). And the programs "Development and Applications of sensor network applied to monitor bloom of blue-green algae in Taihu Lake" (2010ZX03006-006).

\section{References}

1. Xu, H.: Development of fishery equipment and engineering science in China (2005-2006). J. Fishery Modernization 34, 1-8 (2007) (in Chinese)

2. Fan, X.-g.: Analysis of aquaculture development of 2004 and prospect of 2005. J. China Aquaculture 1, 3-5 (2005) (in Chinese)

3. Fu, X.-s., Zhang, P., Zhang, H.-y., Wu, J.-h.: Real-time Meter Measuring pH, Conductivity and Turbidity for Groundwater. J. Instrument Technique and Sensor 11, 14-19 (2004) (in Chinese)

4. Gong, Y.-m., Wang, J.-j., Xiao, D.-y.: The design of intelligent industrial acidity meter based on embedded PC. J. Process Automation Instrumentation 8, 25-28 (2003) (in Chinese)

5. Chen, M.-w., Zhou, X.-p., Liu, X.-n.: Design of ATmega16 based on-line measurement and control instrument for $\mathrm{pH}$ value. Journal of Southeast University (Natural Science Edition) 11, 77-78 (2005) (in Chinese)

6. Liu, X.-q., Sun, Y.-k., Zhao, D.-a., Cheng, L., Zhao, B.-h., Qin, Y., Xu, W.-z.: Research on intelligent supervising and control system about factors of aquiculture. J. Chinese Journal of Scientific Instrument 27, 527-530 (2006) (in Chinese)

7. Yang, S.-y., Yin, J.-p., Zhong, C.-q., Zhang, L.-y., Li, Z.-h.: Study and Implementation of $\mathrm{pH}$ Intelligent Measuring Technology. J. Instrument Technique and Sensor 10, 7-10 (2004) (in Chinese)

8. Camara, L.: Automatic generation of intelligent instruments for IEEE 1451. J. Measurement 35, 3-9 (2004)

9. Institute of Electrical, Electronics Engineers, Inc. IEEE Standards for a Smart Transducer Interface for Sensors and Actuators-Transducer to Microprocessor Protocols and Transducer Electronic Data Sheet (TEDS) Formats, New York, USA Institute of Electrical, Electronics Engineers, Inc. (1997)

10. Song, G., Song, A., Huang, W.: Distributed measurement system based on networked smart sensors with standardized interfaces. J. Sensors and Actuators A 120, 147-153 (2005) (in Chinese)

11. Pardo, A., Camara, L.: Gas measurement systems based on IEEE1451.2 standard. J. Sensors and Actuators B 116, 11-16 (2006)

12. Miroslav, S., Radimir, V.: Integrated Smart Sensor Networking Framework for Sensor-based Appliances. IEEE Sensor Journal 5, 579-586 (2003) 